Supplement of Earth Syst. Dynam., 8, 677-696, 2017

https://doi.org/10.5194/esd-8-677-2017-supplement

(C) Author(s) 2017. This work is distributed under

the Creative Commons Attribution 3.0 License.

(c) (i)
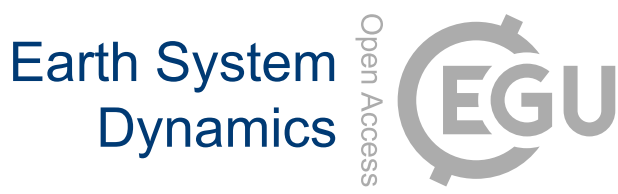

Supplement of

\title{
Multivariate anomaly detection for Earth observations: a comparison of algorithms and feature extraction techniques
}

Milan Flach et al.

Correspondence to: Milan Flach (milan.flach@bgc-jena.mpg.de)

The copyright of individual parts of the supplement might differ from the CC BY 3.0 License. 


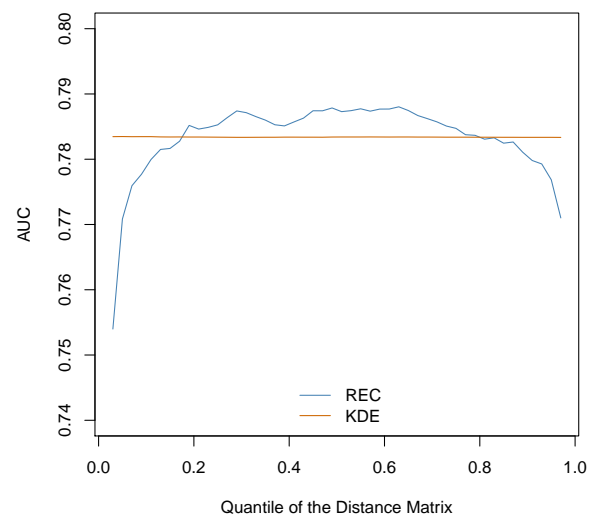

Figure S1. We test different choices of $\sigma$ (Recurrences $(R E C)$ ) or $\varepsilon$ (for Kernel Density Estimation $(K D E)$ ) in a small simulation (500 repetitions) trying to detect a BaseShift. $\sigma$ (or $\varepsilon$, respectively) is varied between the 0.05 and 0.95 quantile of the distribution of values of the distance matrix. The Area Under the receiver operator characteristics Curve $(A U C)$ is computed for each parameterization. Results exhibit constant $A U C$ values for $K D E$ within the testing range of $\sigma$. In contrast $R E C$ is more sensitive to the choice of $\varepsilon$, although it might yield slightly higher $A U C$ values in case of optimal chosen $\varepsilon$. 

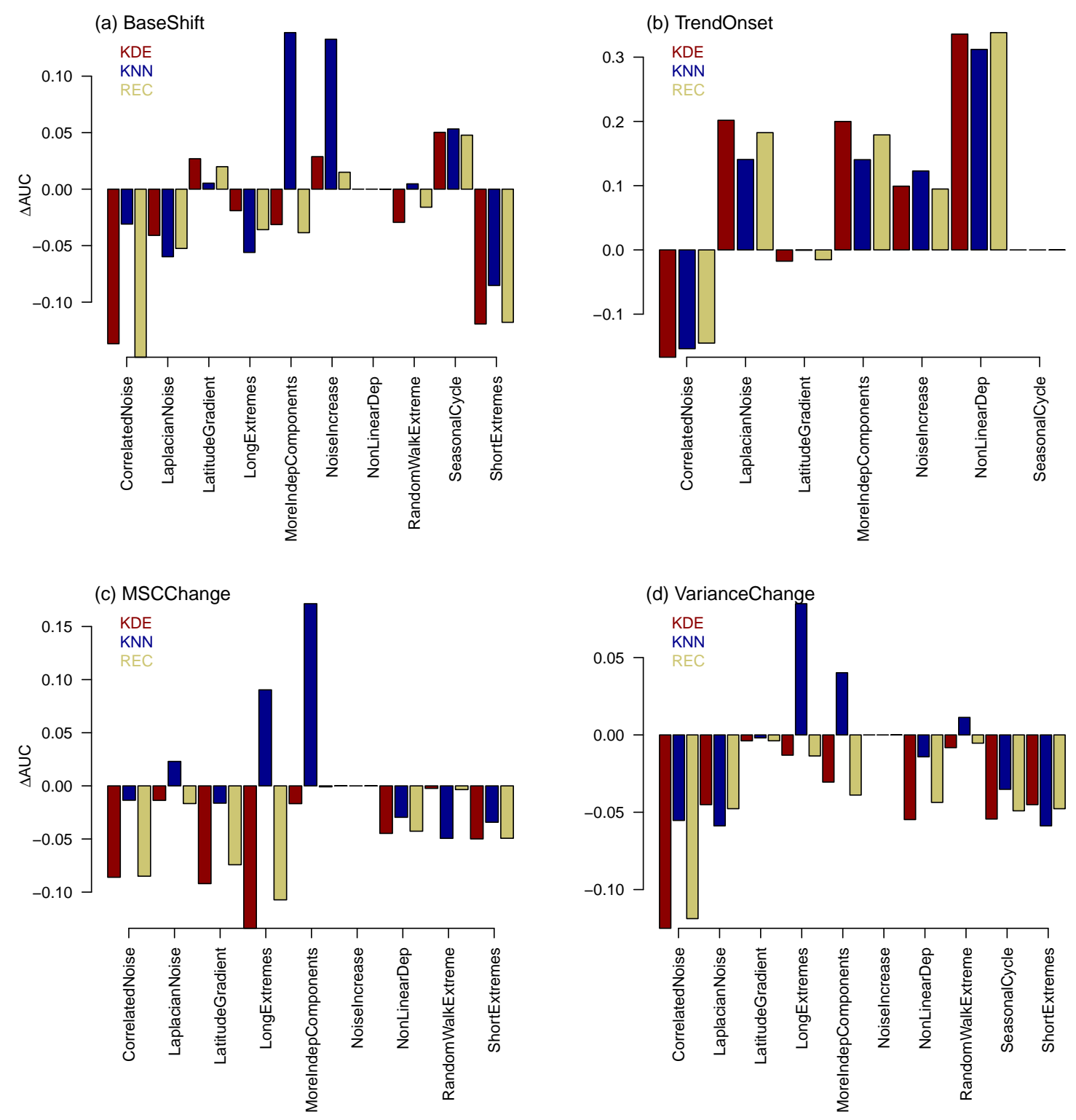

Figure S2. Effect of different data properties on the 3 best detection algorithms (KDE, REC, KNN-Gamma) presented as AUC difference to the UNIV control for the event types (a-d). Details for each algorithm reveal that KNN-Gamma is often less affected by 'difficult' data properties like CorrelatedNoise or MoreIndepComponents, i.e. KNN-Gamma is more adaptive than the other two algorithms. 